\title{
Enhancing quantum Fisher information by utilizing uncollapsing measurements
}

\author{
Juan $\mathrm{He}^{1 \dagger}$, Zhi-Yong Ding ${ }^{1}$, Liu Ye ${ }^{2}$ \\ 1. School of Physics \& Electronics Science, Fuyang Normal College, Fuyang, 236037, People's Republic of China \\ 2. School of Physics \& Material Science, Anhui University, Hefei. 230039, People's Republic of China
}

\begin{abstract}
As an indicator of estimation precision, quantum Fisher information (QFI) lies at the heart of quantum metrology theory. In this work, an effective scheme for enhancing QFI is proposed by utilizing quantum uncollapsing measurements. Two kinds of strategies for the arbitrary two-qubit pure state with weight parameter and phase parameter are implemented under different situations, respectively. We derive the explicit conditions for the optimal measurement strengths, and verify that the QFI can be improved quite well. Meanwhile, due to the relation of quantum correlation and QFI, the maximal value of QFI associated with phase parameter for pure state is always equal to 1 . It is worth noting that the optimal measurement strength is only related to the weight parameter, as uncollapsing measurements operation does not induce any disturbance on the value of phase parameter. The scheme also can be extended to improve the parameter estimation precision for an $\mathrm{N}$-qubit pure state. In addtion, as an example, the situation of an arbitrary single-qubit state under amplitude damping channel is investigated. It is shown that our scheme also works well for enhancing QFI under decoherence.
\end{abstract}

Keywords: Quantum Fisher information; parameter estimation; quantum uncollapsing measurements

PACS numbers: 03.67.-a, 06.20.-f, 03.65.Ta

\section{Introduction}

As one of the most fundamental missions in information theory, parameter estimation has been generalized to quantum field [1-3] due to the rapid development of quantum information theory and quantum technology. Fisher information, originally introduced by Fisher [4], plays a

\footnotetext{
$\dagger$ Corresponding author: juanhe78@163.com
} 
centric role in both statistical theory and quantum metrology. It quantifies the information that one can draw an associated parameter from a probability distribution. Generally speaking, the larger the value of Fisher information, the higher parameter precision we can estimate. Quite recently, quantum Fisher information $(\mathrm{QFI})$, which is the extension of Fisher information in quantum regime, has attracted more and more attention [5-16]. Since QFI can capture the immanent sensitivity of the given system in regard to the change of certain specific parameters, it generally unveils more intricate performance than other correlation measures such as entanglement and quantum discord. Not only is it an available tool to evaluate the accuracy limits of quantum measurements but also it can accomplish various quantum information tasks in entanglement detect [7], investigation of uncertainty relations [17] and so on. Furthermore, as a crucial measure of information content for quantum state, QFI can describe the sensitivity of the state about disturbance of the parameter. Hence, how to enhance the precision of parameter estimation is the primary topic in quantum metrology, meanwhile it is of significant applications in quantum technology such as measurement of gravity accelerations [18], quantum frequency standards [19], clock synchronization[20], etc.

On the other hand, considerable attention [21-27] has been concentrated on uncollapsing measurement, since it can protect entanglement from decoherence and have been experimentally realized in solid system [28], linear optic devices [29,30], and superconducting phase qubits [31,32]. It also has been found to be useful in entanglement amplification [33]. Essentially, the so-called uncollapsing measurement can be seen as generalizations of Von Neumann measurements and associated with a positive-operator valued measure (POVM). The corresponding maps of quantum uncollapsing measurement with strength $m$ and measurement reversal with strength $r$ on one qubit in the computational basis $\{|0\rangle,|1\rangle\}$ can be described as a non-unitary quantum operation $M=\left(\begin{array}{cc}1 & 0 \\ 0 & \sqrt{1-m}\end{array}\right)$ and $R=\left(\begin{array}{cc}\sqrt{1-r} & 0 \\ 0 & 1\end{array}\right)$, respectively.

In this article, we focus our attention on probing an efficient way to improve QFI via uncollapsing measurements. Here the parameterized two-qubit pure state is considered and two strategies can be effectively implemented for different initial state. The merit of our scheme is that the uncollapsed state caused by pre-uncollapsing measurements can be certainly revived to the initial case in probability via suitable post-reversal measurements. It is worth mentioning that the 
scheme can be expanded to the case of an $N$-qubit pure state and open system. This paper is organized as follows. In section 2, QFI is reviewed briefly. In section 3, we propose a method for enhancing QFI via uncollapsing measurements in detail. In section 4, we give a simple example to show our scheme also works well for open system. Finally, a summary is given in section 5 .

\section{Quantum Fisher information}

We briefly review parameter estimation theory and provide the analytical computational method of QFI in this subsection. Assuming an $N$-dimensional quantum state $\rho_{\eta}$ which depends on an unknown parameter $\eta$, one could implement a set of quantum measurements $\{M(\varsigma)\}$ to extract information about $\eta$ from $\rho_{\eta}$. According to the classical estimation theory, the quality of any measurement can be evaluated by Fisher information $[1,2]$,

$$
f_{\eta}^{\prime}=\int d \varsigma p(\varsigma \mid \eta)\left[\frac{\partial \ln p(\varsigma \mid \eta)}{\partial \eta}\right]^{2}
$$

where $p(\varsigma \mid \eta)=\operatorname{Tr}\left[M(\varsigma) f_{\eta}\right]$ is the conditional probability of acquiring the measurement result $\varsigma$ when the value of the parameter is $\eta$.

By optimizing over all possible measurements, QFI [34] can be defined as

$$
f_{\eta}=\max _{M(\varsigma)} f_{\eta}^{\prime}=\operatorname{Tr}\left(\rho_{\eta} L_{\eta}^{2}\right),
$$

where $L_{\eta}$ is the so-called symmetric logarithmic derivative (SLD) and satisfies

$$
\frac{\partial \rho_{\eta}}{\partial \eta}=\frac{1}{2}\left(\rho_{\eta} L_{\eta}+L_{\eta} \rho_{\eta}\right)
$$

here, the complete set of eigenvectors of $L_{\eta}$ composes the optimal POVM to reach the QFI. Obviously, the calculation of QFI can be an easy task if the explicit form of the Hermitian operator $L_{\eta}$ is known. However, unfortunately, only for several special cases, the analytical solution of $L_{\eta}$ is simply found by utilizing some mathematical tricks [35-38].

From the perspective of geometry, owing to the spontaneous relation between QFI and Bures distance, the expression of QFI also can be directly acquired by utilizing the following formula

$$
\mathrm{D}_{B}^{2}\left(\rho_{\eta}, \rho_{\eta+d \eta}\right)=\frac{1}{4} f_{\eta} d \eta^{2}
$$


where the Bures distance can be read as

$$
\mathrm{D}_{B}(\rho, \sigma)=\sqrt{2}\left(1-\operatorname{Tr} \sqrt{\rho^{1 / 2} \sigma \rho^{1 / 2}}\right)^{1 / 2}
$$

That is, if we can get the explicit form of Bures distance of the corresponding states, then the formula of QFI can be obtained straightforwardly by utilizing this relation. Although this expression has already been exploited in several situations [39,40], nevertheless, it cannot be ignored is that this strategy is somewhat difficult to use especially in multipartite system.

On the other hand, by employing the spectral decomposition $\rho_{\eta}=\sum_{i=1}^{K} q_{i}\left|\phi_{i}\right\rangle\left\langle\phi_{i}\right|\left(q_{i}\right.$ and $\left|\phi_{i}\right\rangle$ are the eigenvalue and eigenvector of $\rho_{\eta}$ ), in terms of the subset $\left\{\left|\phi_{i}\right\rangle\right\}$ with $q_{i} \neq 0$, QFI has a more expedient way that can be expressed as [41, 42]

$$
f_{\eta}=f_{C}+f_{Q}
$$

where

$$
f_{C}=\sum_{i=1}^{K} \frac{\left(\partial_{\eta} q_{i}\right)^{2}}{q_{i}}
$$

is the classical Fisher information for probability distribution,

$$
f_{Q}=\sum_{i=1}^{K} q_{i} f_{\eta, i}-\sum_{i \neq j}^{K} \frac{8 q_{i} q_{j}}{q_{i}+q_{j}}\left|\left\langle\phi_{i} \mid \partial_{\eta} \phi_{j}\right\rangle\right|^{2}
$$

is the quantum contribution determined by both eigenvalues and eigenvectors,

and

$$
f_{\eta, i}=4\left(\left|\left\langle\partial_{\eta} \phi_{i} \mid \partial_{\eta} \phi_{i}\right\rangle\right|-\left|\left\langle\phi_{i} \mid \partial_{\eta} \phi_{i}\right\rangle\right|^{2}\right)
$$

is QFI of the pure state $\left|\phi_{i}\right\rangle$. Since this method is more intuitive and accessible, we here utilize Eqs.(6)- (9) to capture QFI.

\section{QFI amplification via uncollapsing measurements}

In this section, we study how employ quantum uncollapsing measurements and measurement reversals to enhance QFI for two-qubit entangled state. 


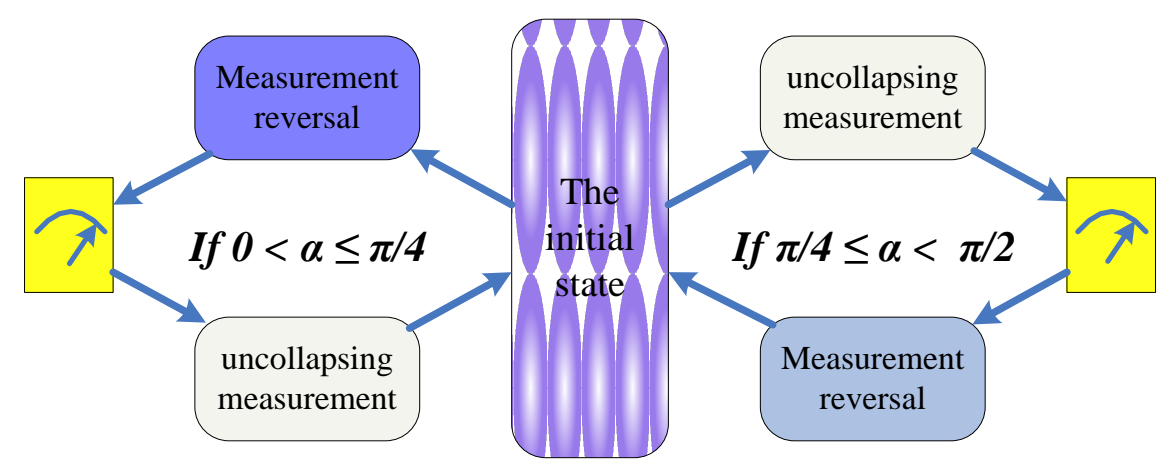

Fig.1. (Color online) The process for improving QFI by means of uncollapsing measurements and measurement reversals.

We assume that a pair of particles A and B are initially in an arbitrary two-qubit pure state, which has the form

$$
|\psi\rangle=\cos \alpha|00\rangle+e^{i \beta} \sin \alpha|11\rangle
$$

where the unknown ingredients $\alpha$ and $\beta$ could be regarded as weight and phase parameters respectively. The relevant two-qubit uncollapsing measurements and measurement reversals operation can be described by $\rho^{(1)}=M^{\prime} \rho M^{\prime+}$ and $\rho^{(2)}=R^{\prime} \rho R^{\prime+}$, here $M^{\prime}=\left(\begin{array}{cc}1 & 0 \\ 0 & \sqrt{1-m_{1}}\end{array}\right) \otimes\left(\begin{array}{cc}1 & 0 \\ 0 & \sqrt{1-m_{2}}\end{array}\right)$ and $R^{\prime}=\left(\begin{array}{cc}\sqrt{1-r_{1}} & 0 \\ 0 & 1\end{array}\right) \otimes\left(\begin{array}{cc}\sqrt{1-r_{2}} & 0 \\ 0 & 1\end{array}\right)$. For simplicity, one can assume the measurement strength $m_{1}=m_{2}=m$ and $r_{1}=r_{2}=r$. The specific process for enhancing QFI by means of uncollapsing measurements and measurement reversals is shown in Fig.1. In the light of different range of weigh parameter, two symmetrical strategies can be implemented, which is based on the fact that uncollapsing measurements can be reversed, i.e., the uncollapsed state caused by pre-uncollapsing measurements can be certainly revived to the initial case in probability via suitable post-reversal measurements. The detailed course will be discussed as follows.

\subsection{Via uncollapsing measurements}

If the weight parameter satisfies the condition $\pi / 4<\alpha<\pi / 2$, performing uncollapsing measurements on two qubits simultaneously, one can obtain the state 


$$
\rho^{(1)}=\frac{1}{\cos ^{2} \alpha+(1-m)^{2} \sin ^{2} \alpha}\left(\begin{array}{cccc}
\cos ^{2} \alpha & 0 & 0 & e^{i \beta}(1-m) \cos \alpha \sin \alpha \\
0 & 0 & 0 & 0 \\
0 & 0 & 0 & 0 \\
e^{-i \beta}(1-m) \cos \alpha \sin \alpha & 0 & 0 & (1-m)^{2} \sin ^{2} \alpha
\end{array}\right)
$$

We only need the nonzero eigenvalues based on Eq.(6)

$$
\lambda=1,
$$

And the corresponding normalized eigenvector is

$$
\left|\phi^{(1)}\right\rangle=\frac{1}{\sqrt{\left(\frac{\cot \alpha}{1-m}\right)^{2}+1}}\left\{\frac{e^{i \beta} \cot \alpha}{1-m}, 0,0,1\right\} .
$$

Firstly, note that the only nonzero eigenvalue is a constant, the classical Fisher information can be expressed as $f_{C}=0$ and $\sum_{i \neq j}^{K} \frac{8 q_{i} q_{j}}{q_{i}+q_{j}}\left|\left\langle\phi_{i} \mid \partial_{\eta} \phi_{j}\right\rangle\right|^{2}=0$.

The QFI with respect to weight parameter $\alpha$ can be achieved by $f_{\alpha}=4\left(\left|\left\langle\partial_{\alpha} \phi \mid \partial_{\alpha} \phi\right\rangle\right|-\left|\left\langle\phi \mid \partial_{\alpha} \phi\right\rangle\right|^{2}\right)$.

Owing to the facts that $\left|\partial_{\alpha} \phi\right\rangle=-\frac{e^{i \beta}(1-m)^{2} \csc ^{2} \alpha}{3}|00\rangle+\frac{(1-m) \cot \alpha \csc ^{2} \alpha}{3}|11\rangle$ and

$$
\left[(1-m)^{2}+\cot ^{2} \alpha\right]^{\frac{3}{2}} \quad\left[(1-m)^{2}+\cot ^{2} \alpha\right]^{\frac{3}{2}}
$$

$\left|\left\langle\phi \mid \partial_{\alpha} \phi\right\rangle\right|=0$, QFI of the weight parameter can be read as

$$
f_{\alpha}^{(1)}=4 \lambda\left|\left\langle\partial_{\alpha} \phi \mid \partial_{\alpha} \phi\right\rangle\right|=\frac{4(1-m)^{2} \csc ^{4} \alpha}{\left[(1-m)^{2}+\cot ^{2} \alpha\right]^{2}} .
$$

Similarly, through some calculation, we also can obtain the QFI for phase parameter $\beta$

$$
f_{\beta}^{(1)}=\frac{4(1-m)^{2} \cot ^{2} \alpha}{\left[(1-m)^{2}+\cot ^{2} \alpha\right]^{2}}
$$

It is not difficult to check that $f_{\alpha}=4$ and $f_{\beta}=\sin ^{2} 2 \alpha$ without any uncollapsing measurement, and both of the $f^{(1)}{ }_{\alpha}$ and $f^{(1)}{ }_{\beta}$ are depend on the value of $\alpha$, while have nothing to do with parameter $\beta$. To illustrate the issue clearly, the contours of QFI $f_{\alpha}^{(1)}$ and $f_{\beta}^{(1)}$ dependence of $\alpha$ and $m$ are plotted in Fig.2 and Fig.3. It is obvious that the QFI is indeed enhanced by utilizing uncollapsed measurements. What's more, when the parameter $\alpha$ lies in the interval 
$(\pi / 4, \pi / 2)$, the variation trend of $f_{\alpha}^{(1)}$ and $f_{\beta}^{(1)}$ is the same, that is, firstly increases and then decreases with the uncollapsing measurement strength $m$ growing, and the value of $m$ relating to the maximal QFI increases as the weigh parameter $\alpha$ increases. However, unlike $f_{\beta}^{(1)}$, which has a fixed maximum, the maximum value of $f_{\alpha}^{(1)}$ increases as $\alpha$ increases monotonously. This indicates that for the weight parameter, the effectiveness of our strategy is more pronounced in improving the QFI for those initial states with greater proportion of the ground states in this case.

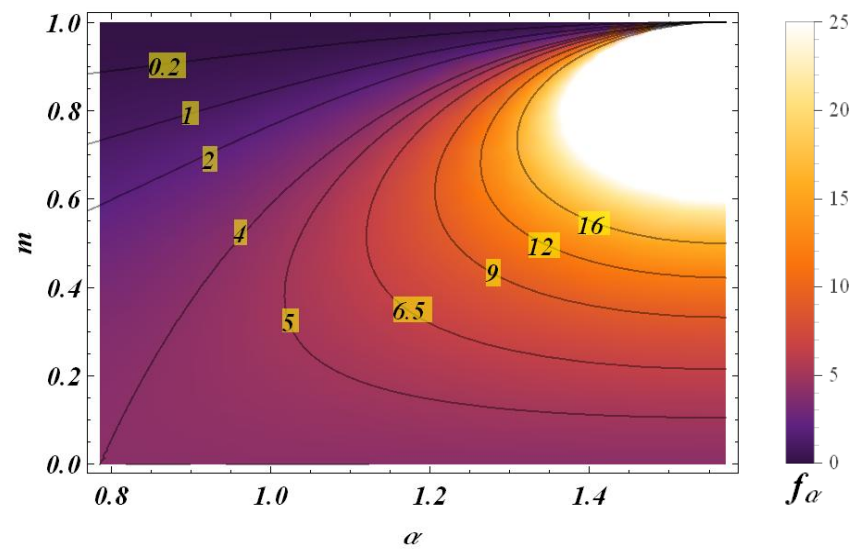

Fig.2. (Color online) The contour plot of QFI $f_{\alpha}$ as a function of the weight parameter $\alpha$ and uncollapsing measurement strength $m$.

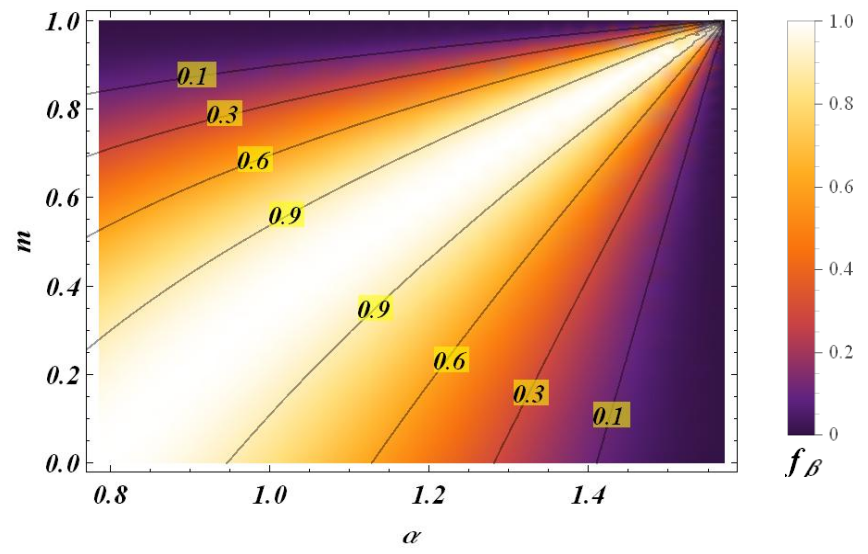

Fig.3. (Color online) The contour plot of QFI $f_{\beta}$ as a function of the weight parameter $\alpha$ and uncollapsing measurement strength $m$. 
In addition, the optimal uncollapsing measurement strength that makes $f_{\alpha(\beta)}^{(1)}$ maximum can be determined from the following conditions

$$
\frac{\partial f^{(1)} \alpha(\beta)}{\partial m}=0 \text { and } \frac{\partial^{2} f^{(1)} \alpha(\beta)}{\partial^{2} m}<0
$$

The corresponding maximal $f_{\alpha}^{(1)}$ and $f_{\beta}^{(1)}$ are $4 \csc ^{2} 2 \alpha$ and 1 , respectively, with the same optimal condition $m=1-\cot \alpha$. Interestingly, the optimal measurement strength is actually independent of phase parameter, but it is related to weigh parameter. Besides, we notice that for the given optimal $m$, the maximum of QFI $f_{\beta}$ is always equal to 1 whatever the initial entangled state is. The underlying reason is that the measurement operation does not cause any disturbance to the value of phase parameter, and all different initial entangled states will transform into a same result (i.e., $\left.\frac{1}{\sqrt{2}}\left(|00\rangle+e^{i \beta}|11\rangle\right)\right)$ when optimal uncollapsing measurement is performed. On the other side, owing to the quantum Cramér-Rao inequality (QCR) [34,43-45], QFI is regarded as the pivotal quantity to characterize the eventual precision in parameter estimation schemes. The well-known QCR inequality is shaped as $\left(\Delta_{\eta}\right)^{2} \geq \frac{1}{\operatorname{Pf}(\eta)}$, where $\left(\Delta_{\eta}\right)^{2}$ refers to the covariance matrix of the unbiased estimator $\hat{\eta}$ and $P$ is the number of measurements. Specially, for two-qubit pure state, the relation between QFI with respect to phase and quantum correlation can be depicted as $\frac{1}{\left(P \Delta_{\beta}\right)^{2}} \leq f(\beta)=2 Q\left(\rho_{A B}\right)$ [45] (noting that quantum correlation $Q\left(\rho_{A B}\right)$ is reduced to entanglement for pure state and the maximum of $Q\left(\rho_{A B}\right)$ is equal to 0.5 with setting $\left.P=1\right)$. It provides a boundary of $\Delta_{\beta}$ and also verifies that the maximal value of $f_{\beta}$ for pure state is always equal to 1 .

\subsection{Via quantum measurement reversals}

In the case of $0<\alpha<\pi / 4$, the so-called measurement reversals is operated on two qubits simultaneously and the state will evolve to 


$$
\rho^{(2)}=\frac{1}{(1-r)^{2} \cos ^{2} \alpha+\sin ^{2} \alpha}\left(\begin{array}{cccc}
(1-r)^{2} \cos ^{2} \alpha & 0 & 0 & e^{i \beta}(1-r) \cos \alpha \sin \alpha \\
0 & 0 & 0 & 0 \\
0 & 0 & 0 & 0 \\
e^{-i \beta}(1-r) \cos \alpha \sin \alpha & 0 & 0 & \sin ^{2} \alpha
\end{array}\right)
$$

The only nonzero eigenvalue and the corresponding normalized eigenvector are $\lambda=1$ and $\left|\phi^{(2)}\right\rangle=\frac{1}{\sqrt{(1-r)^{2} \cot ^{2} \alpha+1}}\left\{e^{i \beta}(1-r) \cot \alpha, 0,0,1\right\}$, respectively.

Through mathematical calculations, the QFI associated with $\alpha$ and $\beta$ can be expressed by

$$
f_{\alpha}^{(2)}=\frac{4(1-r)^{2} \csc ^{4} \alpha}{\left[1+(1-r)^{2} \cot ^{2} \alpha\right]^{2}},
$$

and $f_{\beta}^{(2)}=\frac{4(1-r)^{2} \cot ^{2} \alpha}{\left[1+(1-r)^{2} \cot ^{2} \alpha\right]^{2}}$.

Similarly, from the following formulas

$$
\frac{\partial f_{\alpha(\beta)}^{(2)}}{\partial r}=0 \text { and } \frac{\partial^{2} f_{\alpha(\beta)}^{(2)}}{\partial^{2} r}<0 .
$$

The optimal measurement strength can be obtained that $r=1-\tan \alpha$. The $f_{\alpha}^{(2)}$ and $f_{\beta}^{(2)}$ as function of weight parameter and measurement strength is plotted in Fig.4. From this figure, it is easy to find that the contour of two different cases is symmetric. Like the above case, the variation trends of $f_{\alpha}$ and $f_{\beta}$ in the corresponding chosen range are the same, i.e., firstly increases and then decreases with the measurement strength $r$ growing. Moreover, the maximal QFI of $\alpha$ and $\beta$ remain $4 \csc ^{2} 2 \alpha$ and 1 , respectively.
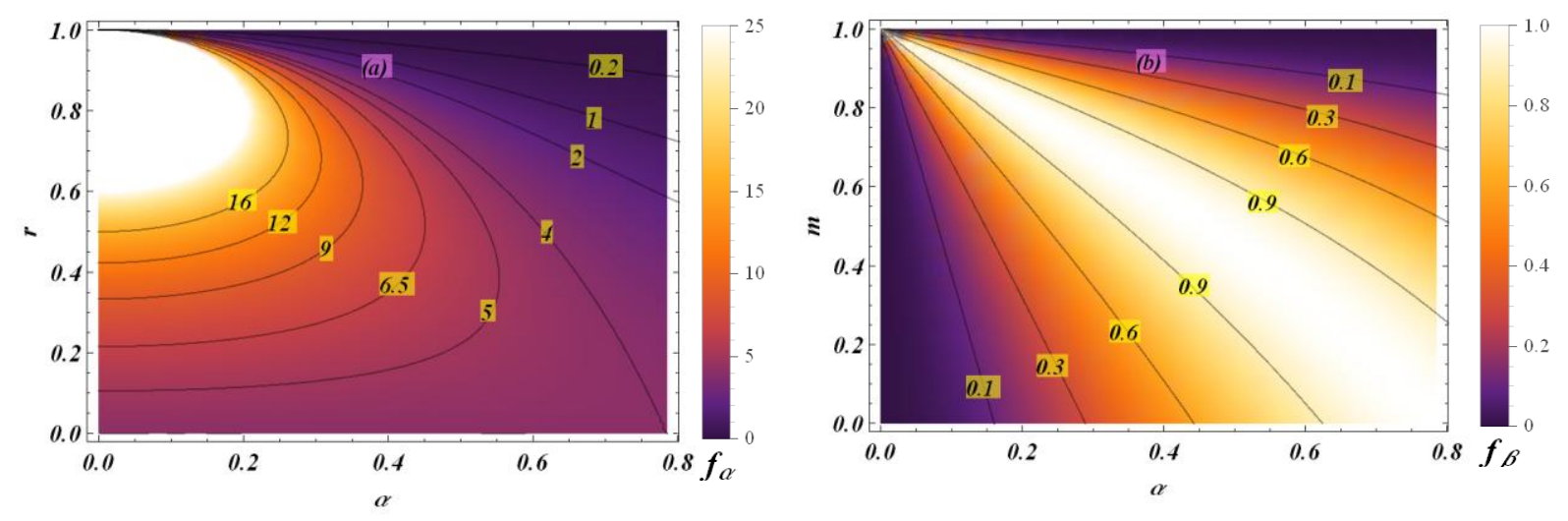
Fig.4. (Color online) The contour plots of QFI $f_{\alpha}(a)$ and $f_{\beta}(b)$ vs the weight parameter $\alpha$ and pre-measurement strength $r$.

Further study tell us the reason that QFI can be improved is that the uncollapsing measurements can change the proportion between ground state and excited state. By means of quantum uncollapsing measurements or measurement reversals, a prior reduction of the excited-state or ground-state component weight makes the system more pronounced in improving QFI. After the parameter estimation, a proper measurement reversals or post-weak measurements is needed. The corresponding success probability is

$$
S p^{(1)}=S p^{(2)}=(1-r)^{2} \cos ^{2} \theta+(1-m)^{2} \sin ^{2} \theta
$$

It is worth emphasizing that the disturbed state caused by pre-measurements can be revived with some probability when the measurement reversal strength is equal to that of uncollapsing measurement, namely, $m=r$. In this case, the success probability is $S p=(1-m)^{2}$. We note that the success probability of pre-measurement seems to affect the QFI of the given state, however, it is not true, since we can confirm whether the pre-measurements is successful before the parameter estimation. In fact, the impact of performed pre-uncollapsing measurement makes the initial component weight between excited-state and ground-state becomes more balanced. For the situation of more unbalanced initial state, the stronger measurement strength is needed, and it will bring out the smaller success probability.

According to the above discussions, we generalize our investigation in the case of an $N$-qubit system $|\psi\rangle=\cos \alpha|0\rangle^{\otimes N}+e^{i \beta} \sin \alpha|1\rangle^{\otimes N}$. For brevity, we put the detailed calculation process in the Appendix. The result indicates that the scheme is also effective for the larger system.

\section{Improving QFI under decoherence via uncollapsing measurements}

In realistic quantum information processing, the unavoidable interaction of quantum system with surrounding noisy environments can produce decoherence. Generally, this process can be described by a quantum operation on the initial density matrix $\rho$ as

$$
\varepsilon(\rho)=\sum_{i} E_{i} \rho E_{i}^{\dagger}
$$

where $E_{i}$ are the Kraus operators satisfying $\sum_{i} E_{i}^{\dagger} E_{i}=1$. 
The well-known amplitude-damping environment is a typical example, which can be depicted by the spontaneous emission of a photon by a two-level atom into a zero-temperature environment of electromagnetic-field modes. The corresponding Kraus operators are

$$
E_{1}=\left(\begin{array}{cc}
1 & 0 \\
0 & \sqrt{1-p}
\end{array}\right) \text { and } E_{2}=\left(\begin{array}{cc}
0 & \sqrt{p} \\
0 & 0
\end{array}\right)
$$

where $\mathrm{p}$ represents decoherence parameter.

Owing to the complexity of calculation, we here expand the above scheme to improve QFI of single-qubit state from amplitude-damping decoherence. This case consists of prior weak measurement on qubit before the interaction with the noisy channel, subsequent parameter estimation, and the post measurement reversal.

Consider an arbitrary single-qubit state

$$
|\xi\rangle=\cos \theta|0\rangle+e^{i \varphi} \sin \theta|1\rangle
$$

subjected to amplitude-damping environment. Similarly, before the qubit suffered from decoherece, the prior weak mearement with strength $m$ is needed when the weight parameter satisfies the condition $\pi / 4<\theta<\pi / 2$. After undergoing decoherence, one can perform a parameter precision on the state. For this case, the nonzero eigenvalues are

$$
\lambda_{1}=\frac{1}{2}\left[1-\frac{\sqrt{3-2 m+(m-1)^{2}\left(6 p^{2}-6 p+1\right)+\cos 2 \theta\left(2-2(m-1)^{2}(1-2 p)^{2}+m^{2} \cos 2 \theta\right)+2 p(m-1)^{2}(p-1) \cos 4 \theta}}{2-m+m \cos 2 \theta}\right]
$$

and

$$
\lambda_{2}=\frac{1}{2}\left[1+\frac{\sqrt{3-2 m+(m-1)^{2}\left(6 p^{2}-6 p+1\right)+\cos 2 \theta\left(2-2(m-1)^{2}(1-2 p)^{2}+m^{2} \cos 2 \theta\right)+2 p(m-1)^{2}(p-1) \cos 4 \theta}}{2-m+m \cos 2 \theta}\right]
$$
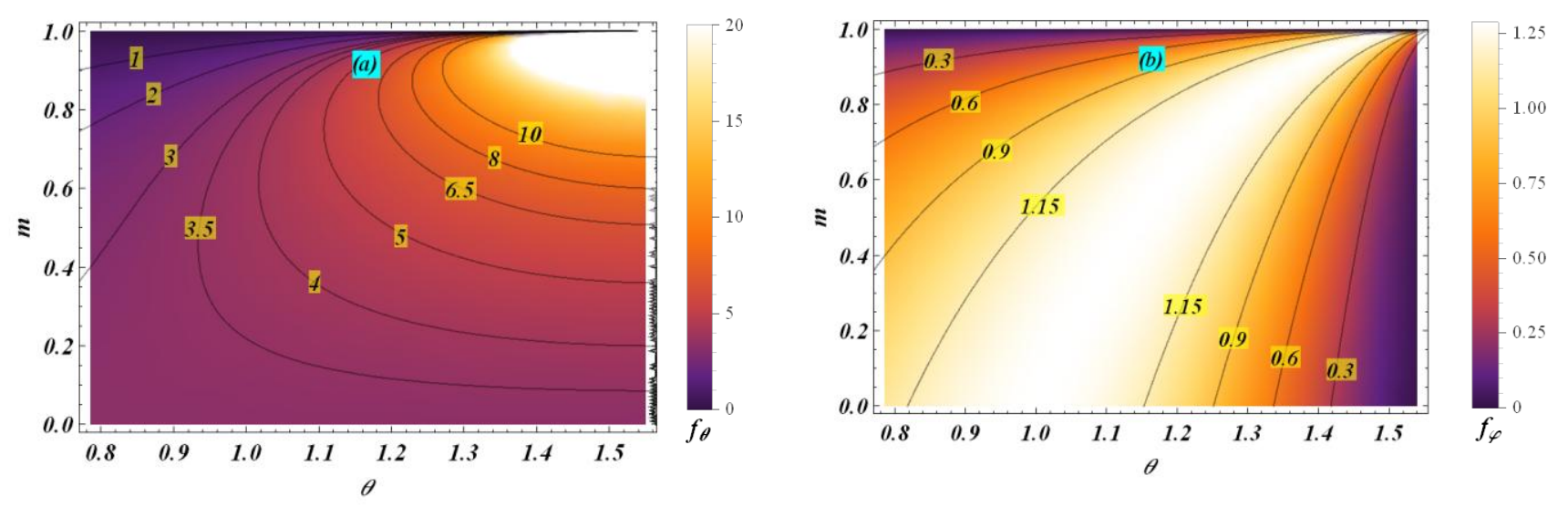
Fig.5. (Color online) The contour plots of QFI $f_{\theta}(a)$ and $f_{\varphi}(b)$ vs the weight parameter $\theta$ and pre-measurement strength $m$.

Accoding to the similar calculation method, we can derive the QFIs of the qubit-state in terms of the weight parameter and phase parameter respectively. Figure 5 displays the variant QFIs of $f_{\theta}$ and $f_{\varphi}$ versus weight parameter $\theta$ and measurement strength $m$ when the decoherence strength set by $p=0.2$.

It is not difficult to find that QFI is indeed enhanced by utilizing uncollapsed measurements. What's more, when parameter $\theta$ lies in the interval $(\pi / 4, \pi / 2)$, the variation trend of $f_{\theta}$ and $f_{\varphi}$ is the same, namely, firstly increases and then decreases with the uncollapsing measurement strength $m$ increases in the effective region, and the value of $m$ correspond to the maximal QFI increases with the weigh parameter $\theta$ growing, which is similar to the above-mentioned in Section 3.

\section{Conclusion}

In summary, we have presented an efficient scheme to improve the QFI of pure state with weight parameter $\alpha$ and phase parameter $\beta$ by means of quantum uncollapsing measurements. The optimal uncollapsing measurement strengths corresponding to the maximal QFI with respect to $\alpha\left(f_{\alpha}\right)$ and $\beta\left(f_{\beta}\right)$ are the same. It is worth mentioning that the optimal measurement strength is actually independent of phase parameter, while related to weight parameter. Futhermore, we have noted that for the given optimal measurement strength, the maximum of QFI for pure state with respect to $\beta\left(f_{\beta}\right)$ is always equal to 1 , whatever the initial entangled state is. For the case of $\pi / 4<\alpha<\pi / 2$, the effectiveness of our scheme is more pronounced in improving the parameter estimation precision for those initial states with greater proportion of the ground states, while for the another case of $0<\alpha<\pi / 4$, the effect is more prominent with greater proportion of the excited state. It is also worth emphasizing that the reason why the QFI of relevant parameters does not involve the success probability of uncollapsing measurements is that we can confirm whether the pre-measurements is successful before the parameter estimation, in spite of the pre-measurement is probabilistic. In addition, our scheme can be expanded to enhance 
the parameter estimation precision for an $\mathrm{N}$-qubit pure state. To verify the scope of this scheme, we investigate, as an example, the situation of an arbitrary single-qubit state under amplitude damping channel. The result has shown that the method is also works for enhancing QFI under decoherence.

\section{Acknowledgment}

This work was supported by the National Science Foundation of China under Grant Nos. 61275119 and 11575001, the Natural Science Research Project of Education Department of Anhui Province of China under Grant KJ2016A547, and also by the Doctoral Foundation of Fuyang Normal University under Grant No. FYNU1602.

\section{Appendix}

Here, we give the detailed calculation process for an $N$-qubit initial system $|\psi\rangle=\cos \alpha|0\rangle^{\otimes N}+e^{i \beta} \sin \alpha|1\rangle^{\otimes N}$. For the case of $\pi / 4<\alpha<\pi / 2$ is discussed. Performing the pre- uncollapsing measurements on $n$ qubits $(n \leq N)$, the state is obtained

$\rho^{(N)}=\frac{1}{\cos ^{2} \alpha+(1-m)^{n} \sin ^{2} \alpha}\left(\begin{array}{cccccccc}\cos ^{2} \alpha & 0 & 0 & \cdots & \cdots & 0 & 0 & e^{i \beta}(1-m)^{n / 2} \cos \alpha \sin \alpha \\ 0 & 0 & 0 & 0 & 0 & 0 & 0 & 0 \\ 0 & 0 & \ddots & 0 & 0 & . & 0 & 0 \\ \vdots & 0 & 0 & 0 & 0 & 0 & 0 & \vdots \\ \vdots & 0 & 0 & 0 & 0 & 0 & 0 & \vdots \\ 0 & 0 & . & 0 & 0 & \ddots & 0 & 0 \\ 0 & 0 & 0 & 0 & 0 & 0 & 0 & 0 \\ e^{-i \beta}(1-m)^{n / 2} \cos \alpha \sin \alpha & 0 & 0 & \cdots & \cdots & 0 & 0 & (1-m)^{n} \sin ^{2} \alpha\end{array}\right)$

The only nonzero eigenvalues is $\lambda=1$ and the corresponding normalized eigenvector is $\left|\phi^{(N)}\right\rangle=\frac{e^{i \varphi} \cot \alpha}{\sqrt{\cot ^{2} \alpha+(1-m)^{n}}}|0\rangle^{\otimes N}+\frac{(1-m)^{n}}{\sqrt{\cot ^{2} \alpha+(1-m)^{n}}}|1\rangle^{\otimes N}$.

Through mathematical calculation, the $\underset{\alpha}{(1) N}$ and $f_{\beta}^{(1) N}$ are boiled down to

$$
f_{\alpha}^{(1) N}=\frac{4(1-m)^{n} \csc ^{4} \alpha}{\left[(1-m)^{n}+\cot ^{2} \alpha\right]^{2}} \text { and } f_{\beta}^{(1) N}=\frac{4(1-m)^{n} \cot ^{2} \alpha}{\left[(1-m)^{n}+\cot ^{2} \alpha\right]^{2}} \text {. }
$$

According to Eqs. (13), the optimal measurement strength is $m=1-\cot ^{2 / n} \alpha$, and the corresponding maximal $f_{\alpha}^{(1) N}$ and $f_{\beta}^{(1) N}$ are $4 \csc ^{2} 2 \alpha$ and 1, respectively. 
Similarly, For the another case, $0<\alpha<\pi / 4$, the only nonzero eigenvalues is $\lambda=1$ and the corresponding normalized eigenvector is

$$
\left|\phi^{(2)}\right\rangle=\frac{e^{i \beta}(1-r)^{n / 2} \cot \alpha}{\sqrt{(1-r)^{n} \cot ^{2} \alpha+1}}|0\rangle^{\otimes N}+\frac{1}{\sqrt{(1-r)^{n} \cot ^{2} \alpha+1}}|1\rangle^{\otimes N} .
$$

The corresponding QFI about parameter $\alpha$ and $\beta$ can be acquired by

$$
f_{\alpha}^{(2) N}=\frac{4(1-r)^{n} \csc ^{4} \alpha}{\left[1+(1-r)^{n} \cot ^{2} \alpha\right]^{2}} \text { and } f_{\beta}^{(2) N}=\frac{4(1-r)^{n} \cot ^{2} \alpha}{\left[1+(1-r)^{n} \cot ^{2} \alpha\right]^{2}}
$$

The optimal measurement strength can be acquired that $r=1-\tan ^{2 / n} \alpha$ by utilizing Eqs. (17), and the corresponding maximal $f_{\alpha}^{(2) N}$ and $f_{\beta}^{(2) N}$ remain $4 \csc ^{2} 2 \alpha$ and 1 , respectively. After parameter estimation an appropriate post-uncollapsing measurement operation would be utilized to revive the disturbed state. The corresponding success probability is

$$
S p_{N}^{(1)}=S p_{N}^{(2)}=(1-r)^{n} \cos ^{2} \theta+(1-m)^{n} \sin ^{2} \theta
$$

It is worth noticing that, like the case of two-qubit state, the disturbed state caused by pre-measurements can be revived with some probability when the pre-measurements strength is equal to that of post-measurements, and the success probability becomes to $S p_{N}=(1-m)^{n}$.

\section{References}

[1] A.S.Holevo, Probabilistic and Statistical Aspects of Quantum Theory (North-Holland, Amsterdam,1982).

[2] C. W. Helstrom, Quantum Detection and Estimation Theory. (Academic Press, New York, 1976).

[3] J. Ma, and X. G. Wang, Phys. Rev. A 80, 012318(2009).

[4] R. A. Fisher, Proc. Cambridge Philos.Soc. 22,700(1925)[reprint in Collected Papers of R.A.Fisher, edited by J.H.Bennett(University of Adelaide Press, South Australia,1972)

[5] R.D.Dobrzański, L.Maccone, Phys. Rev. Lett. 113, 250801 (2014).

[6] K. Berrada, Phys. Rev. A 88,035806 (2013).

[7] N. Li, and S. L. Luo, Phys. Rev. A 88, 014301 (2013).

[8] Y. M. Zhang, X. W. Li, W. Yang, and G. R. Jin, Phys. Rev. A 88, 043832 (2013). 
[9] S. Knysh, V. N.Smelyanskiy, and G.A.Durkin, Phys. Rev. A 83, 021804(R) (2011).

[10] S. S. Pang and T.A. Brun, Phys. Rev. A 90, 022117(2014).

[11] S. Gammelmark, and K. Mølmer, Phys. Rev. Lett. 112, 170401 (2014).

[12] Q.S.Tan, Y.X. Huang, L. M. Kuang, and X.G.Wang, Phys. Rev. A 89, 063604(2014).

[13] S. Abdel-Khaled,Quantum Inf. Process., 12, 3761-3769 (2013).

[14] S. Abdel-Khaled, Opt. Quant. Electron., 46, 1055-1064 (2014).

[15] S. Abdel-Khaled, Ann.Phys., 351, 952-959 (2014).

[16] S. Abdel-Khaled, K. Berrada, A.S.F.Obada, Eur. Phys. J. D. 66, 69 (2012).

[17] Y. Watanabe, T. Sagawa, and M. Ueda, Phys. Rev. A 84, 042121 (2011).

[18] A. Peters, K. Y. Chung, and S. Chu, Nature (London) 400, 849(1999).

[19] S. F. Huelga, C. Macchiavello, T. Pellizzari, et al. , Phys. Rev. Lett. 79, 3865(1997).

[20] R. Jozsa, D. S. Abrams, J. P. Dowling, and C. P. Williams, Phys. Rev. Lett. 85, 2010 (2000).

[21] Q. Q. Sun, M. A. Amri, L. Davidovich, M. S. Zubairy, Phys. Rev. A 82, 052323 (2010).

[22] Y. S. Kim, J. C. Lee, O. K. won, and Y. H. Kim, Nat. Phys. 8, 117 (2012).

[23] A. N. Korotkov and K. Keane, Phys. Rev. A. 81, 040103(R) (2010).

[24] Z. X. Man, Y. J. Xia, and N. B. An, Phys. Rev. A 89, 013852 (2014); J. He, S. Xu,and L. Ye. Physica A. 438, 66 (2015).

[25] S. Ashhab and F. Nori, Phys. Rev. A 82, 062103 (2010).

[26] M. L. Hu and H. Fan, Ann. Phys., 327, 851 (2012); M. L. Hu and D. P. Tian, Ann. Phys., 343, $132(2014)$

[27] K. Wang, X. Zhao, and T. Yu, Phys. Rev. A 89, 042320(2014).

[28] A. N. Korotkov and A. N. Jordan, Phys. Rev. Lett. 97, 166805 (2006).

[29] Y. S. Kim, Y. W. Cho, Y. S. Ra, and Y. H. Kim, Opt. Express 17, 11978(2009).

[30] X. Y. Xu, Y. Kedem, K. Sun, et al., Phys. Rev. Lett. 111, 033604 (2013).

[31] N. Katz, M. Ansmann, R. C. Bialczak, et al., Science 312, 1498 (2006).

[32] J. P. Groen, D. Ristè, L. Tornberg, et al., Phys. Rev. Lett. 111, 090506 (2013).

[33] Y. W. Cheong, and S. W. Lee, Phys. Rev. Lett. 109,150402 (2012).

[34] S. L. Braunstein and C. M. Caves, Phys. Rev. Lett. 72, 3439 (1994).

[35] D. Collins, Phys. Rev. A 87, 032301 (2013).

[36] A. Monras, arXiv:1303.3682. 
[37] S. I. Knysh and G. A. Durkin, arXiv:1307.0470.

[38] Z. Jiang, Phys. Rev. A 89, 032128 (2014).

[39] M. Ahmadi, D. E. Bruschi, N. Friis, C. Sab'in, G. Adesso, and I. Fuentes, arXiv:1307.7082.

[40] O. Pinel, P. Jian, N. Treps, C. Fabre, and D. Braun, Phys. Rev.A 88, 040102(R) (2013).

[41] J. Liu, X.Jing, and X. Wang, Phys. Rev. A 88,042316 (2013).

[42] Y. M. Zhang, X. W. Li, W. Yang, and G. R. Jin, Phys. Rev. A 88,043832 (2013).

[43] U. Dorner, et al., Phys. Rev. Lett. 102,040403(2009).

[44] C. W. Helstrom, Quantum Detection and Estimation Theory (Academic Press, New York, 1976).

[45] C. S. Yu, S. X. Wu, X. G.Wang, X. X. Yi, H. S. Song, EPL, 107, 10007 (2014). 